\title{
Teaching Research in Probability Theory and Mathematical Statistics Modeling Idea
}

\author{
Li Zhang \\ College of mathematics and information, China West Normal University, Nanchong Sichuan, 637000, \\ China
}

Keywords: Mathematical modeling idea, Probability theory, Probability and statistics, Mathematical statistics, Case teaching

\begin{abstract}
Integrating the idea and approach of mathematical modeling into the Probability Theory and Mathematical Statistics can provide new idea for the teaching process, make the relation between theory and practice more closers and motivate students' interest in learning. This paper discusses the necessity and feasibility to integrate mathematical modeling into this course and analyzes the whole teaching process based on teaching cases from different angles.
\end{abstract}

\section{Introduction}

Probability Theory and Mathematical Statistics is one of important core mathematics disciplines under the current background of modern information engineering and it is also the specific method for current social economy study and application. In traditional teaching method, the content of this discipline is boring and rigid in ideas, so it fails to let the students to be enthusiastic in learning. If we attempt to integrate mathematical modeling into the design of the course teaching, it can increase the applicability of this course and make it be more closer to professional background and students' life, not only letting students feel the charm of math, but also letting students improving themselves in active practice and exploration.

\section{Brief Introduction Probability Theory and Mathematical Statistics}

Probability Theory and Mathematical Statistics is a scientific discipline with math as its core to summarize statistical regularity based on random phenomenon. This discipline can reveal the inevitability and contingency of some incidents from the perspective of quantification and discuss the relation between the two. The study in the theory content and summarization of scientific methods based on abstract random incidents is helpful for cultivating students' logical thinking ability to solve practical problems.

Integration of mathematical modeling in the "Probability Theory and Mathematical Statistics" is a more creative and new attempt for teaching, which is based on the characteristics of the actual problem and the law of human production and life and uses abstract methods to extract more specific quantitative mathematical problems for this course, utilizes mathematical thinking to solve and analyze the subjects. And the results can later be used to answer the real questions after its results being interpreted and verified. For example, the problems such as finance and investment, winning the lottery, birthdays and other probability problems in real life can be applied and answered, combing the idea of mathematical modeling with mathematical statistics courses. Some education scholars have pointed out that "the introduction of the mathematical modeling with innovative ideas brings more freshness and creativity for probability theory and mathematical statistics course, if further its exploration, you'll find its practical and far-reaching significance for the course. This is an outstanding attempt to train students' analytical skill in theoretical knowledge and consciousness to solve problems. 
The Necessity and Feasibility of Integrating Mathematical Modelling Idea into Teaching of Probability Theory and Mathematical Statistics

Necessity. First, the discipline of "Probability Theory and Mathematical Statistics" is a comprehensive discipline that integrates rational thinking and observing trials, which bears very rich solving methods for mathematical problems such as the more common model method, construction method, transformation method, quantitative methods, and so on. Especially from the traditional classical concept of mathematical model to today's more popular regression analysis models, mathematical modeling idea can always be integrated into courses of this discipline. Probability theory and mathematical statistics based on mathematical modeling puts more emphasis on the rational and thorough analysis on real data to identify some of the necessary data parameters and testing hypotheses in the courses through mathematical modeling. But in fact, some students were not able to use the basic statistical knowledge in mathematical modeling to analyze the real data, which caused that the regression model constructed by them did not achieve the desired results in forecasting time series, therefore introducing the idea of mathematical modeling into " Probability Theory and Mathematical Statistics " and making it comparable with the theoretical basis of the course based on practice is conducive for improving the value of the theory and practice of the course.

Feasibility. As it is mentioned above, the "Probability Theory and Mathematical Statistics" is quite good at processing quantitative indicators data of random phenomena. Its theoretical approach has been recognized by almost all walks of life in society. For example national welfare lottery, environmental pollution control, corporate investment strategies, and social security and so on all choose to use the knowledge of this discipline. The first step of their practices is to conduct quantitative sampling on the issues and solve the problems after making reasonable examination and judgment, so it is feasible to gradually deepen mathematical modeling idea in this discipline. In addition, the integration of mathematical modeling idea can also allow students to have a deeper understanding of the background and significance of practical application of probability theory and of mathematical statistics, more importantly, it can assist students in combining computer statistical software to carry out practical learning and work, laying a certain foundation of professional mathematical modeling capability for the students engaging in relevant professional in future ${ }^{[1]}$

\section{The Analysis on the Whole Process of Integrating Mathematical Modelling Idea into Teaching of Probability Theory and Mathematical Statistics}

Adopting case method can integrate the idea of mathematical modeling into the teaching of "Probability Theory and Mathematical Statistic ". It mainly uses the idea of mathematical modeling to organize teaching and incorporates the probability theory and mathematical statistics into classroom through the assumption, description, modeling and solving of practical problems, so as to realize analysis and interpretation of each case and summarize every knowledge point that may occur.

Exemplification Analysis of Classical Probability Problems. Birthday problem is one of classic examples of classical probability problems. Teaching and analyzing the relevant knowledge of classical probability for students can deepen their understanding of theoretical knowledge of probability theory. For example, one class has 50 students, of which there is probability that at least two students have the same birthday. In this case, in the "Probability Theory and Mathematical Statistics" course, it assumes that there are total 100 people in a class and all the students' date of birth in Gregorian calendar are recorded on an anonymous note and handed in. 50 groups of students are selected by the computer randomly and the same test is conducted in different classes with 100 people for 8 years in a row, following experimental data will be obtained, as shown in Table 1.

Table 1. The Statistics of the Probability Times of at Least Two Students with Same Birthday from Class Test in 8 Years in A Row

\begin{tabular}{|c|c|c|c|c|c|c|c|c|}
\hline Item & \multicolumn{5}{|c|}{ Class } \\
\hline \multirow{2}{*}{ the time of at least two students with the same birthday } & 1 & 2 & 3 & 4 & 5 & 6 & 7 & 8 \\
\cline { 4 - 9 } & 34 & 37 & 35 & 38 & 36 & 34 & 39 & 35 \\
\hline
\end{tabular}


From Table 1, the probability that at least two students have the same birthday is as high as 71 percent. It can be deduced according to the classical probability calculation:

$$
1-\frac{364 \times 365 \times \ldots 336}{365^{30}} \approx 0.710
$$

A new problem arising from it: If we randomly select 50 group of students' birthday notes and ask students to answer the probability of all students with same birthday. Leaving this problem for students is to further deepen their understanding and flexible application of the knowledge of classical probability. On the other hand, we can also examine the extent of the students' understanding of probability theory in the classroom by drawing of lots such as letting students to consider whether the probability of drawing lots at first and at later are the same. In such discussions, students may yield different results, but its purpose is to guide students to analyze the most abstract core issues in probability theory and mathematical calculations and help them establish such a profound concept of conditional probability. This is model teaching methods based on the use of mathematical modeling thought to convert the actual problem into a probability problem. It is more conducive to the use of probabilistic knowledge to solve related problems, which can be said to be a shortcut for solving problems.

Analysis on Economic Benefit Issues. Probability theory is also in common use in real life. It can economize complex issues in a simple manner. This paper sets up several such economic issues.

Forecast of Natural Disasters

Natural disaster prediction is a very important work, which directly affects the efficiency and safety of people's life and production. It is assumed that there are A, B, C, D four methods, to predict natural disasters that could be adopted. If each of these four methods is used individually for predication, the expenses are as shown in Table 2.

Table 2. Statistics of Probability of Success of Four Methods for Forecasting Natural Disasters and Their Expenses

\begin{tabular}{|c|c|c|c|c|}
\hline \multirow{2}{*}{ Item } & \multicolumn{4}{|c|}{ Forecast Method } \\
\cline { 2 - 5 } & $\mathrm{A}$ & $\mathrm{B}$ & $\mathrm{C}$ & $\mathrm{D}$ \\
\hline \multirow{2}{*}{ probability of success(expense/10,000 RMB) } & 0.8 & 0.7 & 0.6 & 0.5 \\
\cline { 2 - 5 } & 80 & 50 & 20 & 5 \\
\hline
\end{tabular}

In this forecast, you can use a certain kind of individual prediction method and you also can combine several forecasting methods. If the spending cap of forecast cost is 100 million, you should consider which method has the greatest probability of success in predicting natural disasters. Thus, the following calculates the probability of the three purely separate incidents and draw three prediction schemes, while the choice of methods for forecasting combination is: A; A + B; B + C + D, as shown in Table 3.

Table 3. Forecasting Projects and Probability of Success in Forecasting with Combining Forecasting Methods

\begin{tabular}{|c|c|c|c|c|c|c|}
\hline \multirow{2}{*}{ Item } & \multicolumn{6}{|c|}{ Scheme } \\
\hline & A & & $\mathrm{A}+\mathrm{B}$ & & $\mathrm{B}+\mathrm{C}+\mathrm{I}$ & \\
\hline probability of success & 0.8 & $1-(1-0.8)$ & $(1-0.7)=0.94$ & $1-(1-0.7)$ & $(1-0.6)$ & $(1-0.5)=0.94$ \\
\hline
\end{tabular}

After new forecasting method being established based on joint forecasting methods, it is found that the probability is 0.94 , which is higher than the probability of picking one particular scheme. After teachers solve and analyze the problem, you can also ask students to conduct probability and mathematical statistics of self-compiled data so that the issue can be further improved and summarize the experience from compiling questions by oneself. Its purpose is how to get more high predicted probability of success with joint forecasting methods. Through the above-mentioned problem, it can be seen that probability theory and mathematical statistics is rather very effective in the use of economic problems, such as marketing, human resources management, which can be used as material for raising issues. 
Probability Theory Case Analysis based on the Central Limit Theorem - Life Insurance Issues

In the course of "Probability Theory and Mathematical Statistics", the elaboration and analysis of central limit theorem can be derived from mathematical modeling thought. It is also the most critical knowledge of probability theory. Utilizing example analysis to promote the use of the central limit theorem in teaching can also stimulate students' interest in learning probability theory. The following takes the relevant insurance contents of one life insurance company as a case for analysis.

100000 people attended the elderly life insurance of a city's life insurance company and the average annual insurance payment per person is 3,000 Yuan. If the applicant is died within the year, the insurance company should compensate the beneficiary 15,000 Yuan. If the local elderly mortality is 0.05 , what is the probability that the insurance company's annual loss in the elderly life insurance business?

It is assumed that $\mathrm{A}$ is the number of deaths of insured elderly every year in this question, where the binomial distribution theory can be used to make A principle subject to the binomial distribution B (100000,0.05), and np is 100, np (1-p) $=99$.

If the insurance company has the phenomenon of loss, it is must meet:

$$
3000 \times 100000-1000 \mathrm{~A}<0
$$

If $\mathrm{A}>3000$, then it is:

$$
p(A>3000)=1-p(0<\mathrm{A}<3000) \approx 1-\left[\Phi\left(\frac{3000-100}{\sqrt{99}}\right)-\Phi\left(\frac{-100}{\sqrt{99}}\right)\right]=2-2 \Phi(10.0500) \approx 0
$$

Through the above reasoning and calculation, utilizing mathematical modeling thought and the central limit theorem to solve the problem of probability, we can confirm the answers of the insurance company's probability in profit and loss so as to deepen students' impression of learning content, furthermore it also can broaden students' ideas, enhance students' practical computing ability to solve some other practical problems in daily life.

Given that the calculation burden of processing data for problems involved in " Probability Theory and Mathematical Statistics " is rather large, so when teachers explain the data reduction and content analysis in examples, they should combine computer statistical software as much as possible, adopting multimedia way for demonstrating SAS, SPSS and other software functions for students, and optimizing statistical methods and statistical models for some students, which also can clarify the step procedures and results of data processing and statistical. Meanwhile, in the classroom teachers should guide students to combine mathematical modeling idea on their own for thinking solving methods for some probability theory problems and make full use of such data fitting, statistical inference for relevant xperiments, so that teachers can enhance students' application consciousness and ability for the course of "Probability Theory and Mathematical Statistics " ${ }^{[2]}$.

\section{The Relevant Suggestions for Teaching Reforming of Probability Theory and Mathematical Statistics}

\section{Reforming Textbook Content.}

"Probability Theory and Mathematical Statistics" should follow the principle of "application being as the purpose, enough for use being as the scale" for reforming teaching materials, but also at the same time domestic and foreign materials should be borrowed for improving and enriching the content of the course so that students can grasp more relevant knowledge in practical application. Furthermore, the difficulty of the content of textbooks should be reduced and more economic application examples close to daily life should be adopted so as to let students feel the practical application of mathematical modeling idea based on theoretical study. For example, a school complied the application type textbook "Probability Statistics of Economic Applied Mathematics Foundation" for students. The characteristics of the teaching is that it adds one particular economic 
application example that is close to production life after each chapter so that students can find and solve problems through examples after theoretical study, which lays a good solid theoretical foundation for students in future's new content study.

Participating in More Mathematical Modeling Competition. College students mathematical modeling contest was held in the early 1980s in the United States, college students in our country are still strangers to this kind of competition and few students are interested in mathematical modeling, so mathematical modeling courses should be carried out right after students enter universities so as to train their modeling idea and create opportunities to participate in relevant mathematical modeling contest for them, which is also to foster students' mathematical modeling idea from another perspective, thereby it can eliminate theory cognitive obstacles for them learning probability theory and mathematical statistics course in future and develop their modeling thinking.

Reforming of Evaluation Method. Mathematical modeling should be integrated into the evaluation content of "Probability Theory and Mathematical Statistics" course. For example, learning content in classroom accounts for $10 \%$, while homework after class accounts for $20 \%$. The purpose is to reflect students' interest and enthusiasm in learning this course, but also reflects the students to mastery and application knowledge of probability statistics knowledge. So the flexible evaluation mechanism is also an objectively reflection of student's actual ability ${ }^{[3]}$.

Summary: In summary, integrating modeling thought into the specific application of teaching "Probability Theory and Mathematical Statistics" is to dually deepen theory and practice study. It is not just to improve the students' learning ability, but also evaluate teachers' teaching quality. And in the course based on random phenomena statistical regularity, more case analysis are obviously more attractive and convincing for students, therefore such mathematical modeling idea and teaching method should be gradually infiltrated in future's courses so as to keep exploring the innovative idea for teaching and application.

\section{References}

[1] Chen Haiying. Study on Teaching Reform of Integrating Mathematical Modeling Idea into "Probability Theory and Mathematical Statistics". Journal of Hubei University of Economics (Humanities and Social Sciences) ,2014,(9):177-178.

[2] Liu Xiaoge. Initial Study and Exploration on Integrating Integrating Mathematical Modeling Idea into "Probability Theory and Mathematical Statistics". The Science Education Article Collects,2014,(9):177-178.

[3] Gan Jing. Viewing the Teaching of "Probability Theory and Mathematical Statistics" from the Perspective of Mathematical Modeling. Chi Zi,2015,(3):238-238. 